\title{
AN INEQUALITY FOR PROBABILITIES
}

\author{
D. A. DAWSON ${ }^{1}$ AND D. SANKOFF
}

1. The main result and applications. Given a probability measure space $(\Omega, \mathfrak{F}, P)$, let $A_{k} \in \mathfrak{F}, k=1, \cdots, N$. The main result is given in the following theorem.

THEOREM 1.1.

$$
P\left(\bigcup_{k=1}^{N} A_{k}\right) \geqq \frac{\theta B^{2}}{2 \alpha+(2-\theta) B}+\frac{(1-\theta) B^{2}}{2 \alpha+(1-\theta) B}
$$

where $B=\sum_{k=1}^{N} P\left(A_{k}\right), \alpha=\sum_{k=1}^{N} \sum_{l=1}^{k-1} P\left(A_{k} \cap A_{l}\right)$ and $\theta=2 \alpha / B$ $-[2 \alpha / \beta], 0 \leqq \theta<1$.

The proof of Theorem 1.1 is given in $\$ 2$.

Corollary 1. A necessary condition for $P\left(\bigcup_{\mathbf{k}=1}^{N} A_{k}\right)<1$ is that B $<\left(1+(1+8 \alpha)^{1 / 2}\right) / 2$.

Proof. It is easy to verify that if the right-hand side of inequality (1.1) is regarded as a function of $\theta$, then the minimum occurs for $\theta=0$. Hence

$$
P\left(\bigcup_{k=1}^{N} A_{k}\right) \geqq \frac{B^{2}}{2 \alpha+B} .
$$

Therefore if $1>P\left(\cup_{k=1}^{N} A_{k}\right)$, then $1>B^{2} /(2 \alpha+B)$, that is, $\beta^{2}-B$ $-2 \alpha<0$. Hence it is necessary that

$$
B<\left(1+(1+8 \alpha)^{1 / 2}\right) / 2 .
$$

The next application is an elementary proof of the Erdös-Rényi form of the Borel-Cantelli lemma [1, p. 326].

Corollary 2. If $A_{k} \in \mathfrak{F}, k=1,2,3, \cdots$, with $\sum_{k=1}^{\infty} P\left(A_{k}\right)=+\infty$, then $P\left(\bigcup_{k=N}^{\infty} A_{k}\right) \geqq 1 / c$ where

$$
c=\liminf _{n \rightarrow \infty} \frac{\sum_{k=1}^{n} \sum_{l=1}^{n} P\left(A_{k} \cap A_{l}\right)}{\left(\sum_{k=1}^{n} P\left(A_{k}\right)\right)^{2}} .
$$

Received by the editors June 10, 1966.

1 This paper was written in part while the first author was a fellow of the Summer Research Institute of the Canadian Mathematical Congress. 
Proof. By Theorem 1.1

$$
P\left(\bigcup_{k=N}^{M} A_{k}\right) \geqq \frac{\theta}{\frac{2 \alpha_{N M}}{Q_{N M}^{2}}+\frac{(2-\theta)}{B_{N M}}}+\frac{(1-\theta)}{\frac{2 \alpha_{N M}}{B_{N M}^{2}}+\frac{(1-\theta)}{B_{N M}}}
$$

where

$$
\begin{aligned}
2 \alpha_{N M} & =2 \sum_{k=N}^{M} \sum_{l=N}^{k-1} P\left(A_{k} \cap A_{l}\right) \\
& =\sum_{k=N}^{M} \sum_{l=N}^{M} P\left(A_{k} \cap A_{l}\right)-\bigotimes_{N M}
\end{aligned}
$$

and

$$
\bigotimes_{N M}=\sum_{k=N}^{M} P\left(A_{k}\right)
$$

Hence since $\lim _{M \rightarrow \infty} \mathbb{B}_{N M}=+\infty$.

$$
\lim _{m \rightarrow \infty} P\left(\bigcup_{k=N}^{M} A_{k}\right) \geqq \frac{1}{c_{N}}
$$

where

$$
\begin{aligned}
c_{N} & =\liminf _{M \rightarrow \infty} \frac{\sum_{k=N}^{M} \sum_{l=N}^{M} P\left(A_{k} \cap A_{l}\right)}{B_{N M}^{2}} \\
& =c .
\end{aligned}
$$

Corollary 2 can also be proved by the following inequality due to Chung and Erdös [2],

$$
P\left(\bigcup_{k=1}^{N} A_{k}\right) \geqq \frac{B^{2}-\otimes}{2 \alpha} .
$$

(1.2) is easily proved by using Schwarz's inequality applied to indicator functions of sets.

However the inequality of Theorem 1.1 is stronger than (1.2) unless $\left(B^{2}-B\right) / 2 \alpha=1$.

In fact it is easy to verify that

$$
\frac{B^{2}}{2 \alpha+B}>\frac{B^{2}-B}{2 \alpha} \quad \text { if } 0 \leqq \frac{B^{2}}{2 \alpha+B}<1
$$

and 


$$
\frac{B^{2}}{2 \alpha+Q}=\frac{B^{2}-B}{2 \alpha} \quad \text { if } \frac{B^{2}}{2 \alpha+B}=1 \text {. }
$$

It will be shown elsewhere that the lower bound of Theorem 1.1 can be attained so that the inequality cannot be improved.

2. Proof of Theorem 1.1. Given $\left\{A_{1}, \cdots, A_{N}\right\}$ we define $B_{r}$, $r=1, \cdots, N$, to be the set of points in $\bigcup_{k=1}^{N} A_{k}$ which belong to exactly $r$ of the sets $\left\{A_{1}, \cdots, A_{N}\right\}$ and let $a_{r}=P\left(B_{r}\right)$.

Then we have

$$
\begin{gathered}
a_{r} \geqq 0, \quad r=1, \cdots, N, \\
\sum_{k=1}^{N} k a_{k}=\sum_{k=1}^{N} P\left(A_{k}\right)=B,
\end{gathered}
$$

and

$$
\sum_{k=2}^{N} \frac{k(k-1)}{2} a_{k}=\sum_{k=1}^{N} \sum_{l=1}^{k-1} P\left(A_{k} \cap A_{l}\right)=\alpha .
$$

We proceed by finding the minimum of the linear expression

$$
V=P\left(\bigcup_{k=1}^{N} A_{k}\right)=\sum_{k=1}^{N} a_{k}
$$

subject to the constraints (2.1), (2.2) and (2.3). Substituting the expression for $a_{r-1}$ which is obtained by solving (2.2) for $a_{r-1}$ into (2.3), and dividing by $r$ to make the coefficient of $a_{r}$ equal to one we obtain

$$
\begin{gathered}
\frac{-(r-2)}{r} a_{1}+\sum_{k=2}^{N} a_{k}\left\{\frac{k(k-1)}{r}-\frac{k(r-2)}{r}\right\} \\
=\frac{2 \alpha}{r}-\frac{(r-2)}{r} B .
\end{gathered}
$$

Substituting the expression for $a_{r}$ which is obtained by solving (2.3) for $a_{r}$ into (2.2), we obtain

$$
a_{1}+\sum_{k=2}^{N} a_{k}\left\{k-\frac{k(k-1)}{r-1}\right\}=B-\frac{2 \alpha}{r-1} .
$$

Now solving (2.5) for $a_{r}$ and (2.6) for $a_{r-1}$ and substituting the resulting expressions into (2.4) we obtain

$$
\text { (2.7) } V-\frac{2 B}{r}+\frac{2 \alpha}{r(r-1)}=a_{1} \frac{(r-2)}{r}+\sum_{k=2}^{N} \frac{(r-k)(r-k-1)}{r(r-1)} a_{k} \text {. }
$$


We henceforth assume that in equations (2.5), (2.6) and (2.7),

$$
\begin{aligned}
r & =2+[2 \alpha / B] \text { if } 2 \alpha / B \neq(N-1) \\
& =N \quad \text { if } 2 \alpha / \beta=N-1 .
\end{aligned}
$$

Since $2 \alpha \leqq(N-1) \otimes$ it follows that $r \leqq N$.

It then follows that

$$
(r-2) / r \geqq 0
$$

and

$$
(r-k)(r-k-1) / r(r-1) \geqq 0, \quad k=2, \cdots, N .
$$

Since (2.9) and (2.10) imply that the coefficients of the $a_{\kappa}$ in the righthand side of equation (2.7) are nonnegative, the minimal value of $V-2 \beta / r+2 \alpha / r(r-1)$ is zero.

But if we set

$$
\begin{aligned}
a_{r-1} & =ß-2 \alpha /(r-1), \\
a_{r} & =2 \alpha / r-(r-2) \Theta / r, \\
a_{k} & =0, \quad k \neq r-1, r,
\end{aligned}
$$

then equations (2.5) and (2.6) are satisfied and $V-2 \beta / r+2 \alpha / r(r-1)$ $=0$. Moreover (2.8) implies that $a_{r-1} \geqq 0$ and $a_{r} \geqq 0$ so that the set of $a_{n}$ 's given by (2.11) minimizes the expression (2.4) and satisfies all the constraints.

Therefore the minimal value of $V$ is

$$
\begin{aligned}
V & =\frac{2 B}{r}-\frac{2 \alpha}{r(r-1)} \\
& =\frac{2 B^{2}}{2 \alpha+(2-\theta) B}-\frac{2 \alpha B^{2}}{(2 \alpha+(2-\theta) B)(2 \alpha+(1-\theta) B)}
\end{aligned}
$$

where $\theta=2 \alpha / \beta-[2 \alpha / \beta], 0 \leqq \theta<1$. But then

$$
V=\frac{\theta B^{2}}{2 \alpha+(2-\theta) B}+\frac{(1-\theta) B^{2}}{2 \alpha+(1-\theta) B} .
$$

\section{REFERENCES}

1. A. RÉNYI, Wahrscheinlichkeitsrechnung, Veb Deutscher Verlag der Wissenschaften, Berlin, 1962.

2. K. L. Chung and P. Erdös, On the application of the Borel-Cantelli lemma, Trans. Amer. Math. Soc. 72 (1952), 179-186.

MCGILl University 